\title{
CYCLE LENGTH IN A RANDOM FUNCTION
}

\author{
BY \\ P. W. PURDOM AND J. H. WILLIAMS
}

Let $X$ be a finite set of $n$ points and $F_{n}$ be the class of $n^{n}$ functions from $X$ into $X$. For any $f \in F_{n}$ and $x_{0} \in X$, the sequence, $x_{0}, x_{1}=f\left(x_{0}\right), x_{2}=f\left(x_{1}\right), \ldots$, is eventually cyclic, i.e. there exists $J$ and there exists $l$ such that $j>J$ implies $x_{j}=x_{j+l}$. We will call $l$ distinct points $x_{i}, x_{i+1}, \ldots, x_{i+l-1}$ a cycle if $x_{j+1}=f\left(x_{j}\right)(i \leqq j \leqq i+l-2)$ and $f\left(x_{i+l-1}\right)=x_{i}$. Clearly different choices of the starting value, $x_{0}$, may lead to different cycles.

The length of the longest cycle in a function is of interest in the generation of pseudo-random numbers [1]. We consider the expected value of the length and the $m$ th moment of the length of the $i$ th longest cycle where the function $f \in F_{n}$ is selected at random.

Given $f \in F_{n}$, let $Y$ be the subset of $X$ consisting of all the points in cycles; then $f$ restricted to $Y$ is a permutation. Letting $\alpha$ be any characteristic of the cycle structure of a function $f \in F_{n}$ (e.g. $\alpha \equiv$ the longest cycle is of length $l$ ), we first find a formula relating the number of functions with characteristic $\alpha$ to the number of permutations with characteristic $\alpha$. We then use the results of Shepp and Lloyd [2] giving asymptotic expressions for the expected values of the various moments of cycle lengths in permutations to find the asymptotic expressions for these values for functions.

We say that a function $f$ directly connects $x_{i}$ to $x_{j}$ if $x_{j}=f\left(x_{i}\right)$ and that $f$ connects $x_{i}$ to $x_{j}$ if there is a sequence of directly connected points starting with $x_{i}$ going to $x_{j}$. Then the subset, $Y$, consists of just those points that are connected to themselves. We say that a subset $Z \subset X$ is a tree rooted on a point $x_{m}$ if: (1) $x_{m} \in X-Z$, (2) $x \in Z$ implies $x$ is connected to $x_{m}$, and (3) no point in $X-Z$ is connected to a point in $Z$. Clearly any $f \in F_{n}$ connects some of the points into cycles and the remainder of the points into trees rooted on points in cycles.

Let $T(n, m)$ denote the number of ways of connecting $n$ points into trees rooted on $m$ of the $n$ points. Since $C_{n, m}$ is the number of ways the root points may be chosen and $m^{i} T(n-m, i)$ is the number of ways the remaining points may be connected if exactly $i$ of them are directly connected to the $m$ roots, we have the recurrence relation,

$$
T(n, m)=C_{n, m} \sum_{i=0}^{n-m} m^{i} T(n-m, i),
$$

where $T(n, 0)=0$ for $n>0$ and $T(0,0)=1$. The solution to the recurrence relation is

Received by the editors May 18, 1967. 
$T(n, m)=C_{n-1, m-1} n^{n-m}$ which may be verified inductively by applying the binomial theorem in the induction step. If $\alpha$ is some characteristic of the cycle structure of a function, let $P(n, \alpha)$ denote the number of permutations of $n$ points having cycles with characteristic $\alpha$ and $N(n, \alpha)$ denote the number of functions in $F_{n}$ having cycles with characteristic $\alpha$. Then,

$$
N(n, \alpha)=\sum_{i=1}^{n} T(n, i) P(i, \alpha)
$$

since there are $T(n, i) P(i, \alpha)$ ways of having $i$ of the points in cycles, and there may be from 1 to $n$ points in cycles.

Using $\alpha_{l, r}$ to denote the characteristic, "the $r$ th longest cycle is of length $l$ ", we have

$$
N\left(n, \alpha_{l, r}\right)=\sum_{i=l}^{n} T(n, i) P\left(i, \alpha_{l, r}\right)
$$

since $P\left(i, \alpha_{l, r}\right)=0$ for $i<l$. Then, over $F_{n}$ the expected value of the $m$ th moment of the length, $l$, of the $r$ th longest cycle is

$$
E_{P_{n}, r}\left(l^{m}\right)=\frac{\sum_{l=1}^{n} l^{m} N\left(n, \alpha_{l, r}\right)}{\sum_{l=0}^{n} N\left(n, \alpha_{l, r}\right)},
$$

since the $l=0$ term in the numerator contributes nothing for $m>0$. But $\sum_{i=0}^{n} N\left(n, \alpha_{l, r}\right)=n^{n}$ since this is just the number of functions in $F_{n}$. Therefore,

$$
\begin{aligned}
E_{F_{n}, r}\left(l^{m}\right) & =\frac{\sum_{l=1}^{n} l^{m} \sum_{j=l}^{n} T(n, j) P\left(j, \alpha_{l, r}\right)}{n^{n}} \\
& =\frac{\sum_{j=1}^{n} T(n, j) \sum_{l=1}^{j} l^{m} P\left(j, \alpha_{l, r}\right)}{n^{n}} .
\end{aligned}
$$

The number of permutations of $j$ points is $j$ ! ; therefore over all the permutations of $j$ points, $P_{j}$, the expected value of the $m$ th moment of the length, $l$, of the $r$ th longest cycle is

$$
E_{P, r}\left(l^{m}\right)=\frac{\sum_{l=1}^{j} l^{m} P\left(j, \alpha_{l, r}\right)}{j !} .
$$

Therefore,

$$
E_{F_{n}, r}\left(l^{m}\right)=\frac{\sum_{j=1}^{n} T(n, j) j ! E_{P_{j, r}}\left(l^{m}\right)}{n^{n}} .
$$

Shepp and Lloyd [2] show that

$$
E_{P, r}\left(l^{m}\right)=\left(G_{r, m}+\varepsilon_{r, m, j}\right) j^{m},
$$


where

$$
\begin{gathered}
\lim _{r \rightarrow \infty}\left(\varepsilon_{r, m, j}\right)=0, \\
G_{r, m}=\int_{0}^{\infty} \frac{x^{m-1}}{m !} \frac{[E(x)]^{r-1}}{(r-1) !} \exp [-E(x)-x] d x,
\end{gathered}
$$

and

$$
E(x)=\int_{x}^{\infty} \frac{e^{-y}}{y} d y
$$

Also, Shepp and Lloyd [2] show that

$$
E_{P_{j, 1}}(l)=G_{1,1}\left(j+\frac{1}{2}\right)+o(1) .
$$

Therefore

$$
\begin{aligned}
E_{F_{n}, r}\left(l^{m}\right) & =\sum_{j=1}^{n} C_{n-1, j-1} n^{n-j} n^{-n} j ! j^{m}\left(G_{r, m}+\varepsilon_{r, m, j}\right) \\
& =G_{r, m} \sum_{j=1}^{n} \frac{(n-1) ! j^{m+1}}{(n-j) ! n^{j}}+\bar{\varepsilon}_{r, m, n}
\end{aligned}
$$

where

$$
\bar{\varepsilon}_{r, m, n}=\sum_{j=1}^{n} \frac{(n-1) ! j^{m+1}}{(n-j) ! n^{j}} \varepsilon_{r, m, j}
$$

We will let

$$
Q_{n}(k)=\sum_{j=1}^{n} \frac{(n-1) ! j^{k}}{(n-j) ! n^{j}}
$$

and show that

$$
\lim _{n \rightarrow \infty} \bar{\varepsilon}_{r, m, n} / Q_{n}(m+1)=0 .
$$

Given $\delta>0$ we first pick a $k$ such that $\left|\varepsilon_{r, m, i}\right|<\delta$ for $i>k$ (Shepp and Lloyd [2]) and then rewrite our limit as

$$
\lim _{n \rightarrow \infty} \frac{\sum_{j=1}^{k} \frac{(n-1) ! j^{m+1}}{(n-j) ! n^{j}} \varepsilon_{n, m, j}}{Q_{n}(m+1)}+\frac{\sum_{j=k+1}^{n} \frac{(n-1) ! j^{m+1}}{(n-j) ! n^{j}} \varepsilon_{r, m, j}}{Q_{n}(m+1)} .
$$

Then since all terms in $Q_{n}(m+1)$ are positive, the right hand term of $(B)$ is less than $\delta$. Since the first $\sqrt{ } n-1$ terms of $Q_{n}(m+1)$ are always increasing, there exists a $q$ such that

$$
\left(\sum_{j=1}^{k} \frac{(n-1) ! j^{m+1}}{(n-j) ! n^{j}} / Q_{n}(m+1)\right)<\delta
$$

when $n>q$. Letting $K=\max _{1 \leqq i \leqq k}\left|\varepsilon_{r, m, i}\right|$, then when $n>q$, the left hand term of $(B)$ is less than $K \delta$, so that we have (A). Thus,

$$
\lim _{n \rightarrow \infty} \frac{E_{F_{n}, r}\left(l^{m}\right)}{Q_{n}(m+1)}=G_{r, m} .
$$


To calculate $Q_{n}(k)$ for $k \geqq 1$ note that

$$
\begin{aligned}
n Q_{n}(k-1)-Q_{n}(k) & =\sum_{j=1}^{n} \frac{(n-1) !(n-j) j^{k-1}}{(n-j) ! n^{j}} \\
& =-\delta_{k, 1}+\sum_{j=0}^{n} \frac{(n-1) !(n-j) j^{k-1}}{(n-j) ! n^{j}} \\
& =-\delta_{k, 1}+\sum_{j=1}^{n} \frac{n !(j-1)^{k-1}}{(n-j) ! n^{j}} \\
& =-\delta_{k, 1}+\sum_{i=0}^{k-1}(-1)^{k-1-i} C_{k-1, i} \sum_{j=1}^{n} \frac{n ! j^{i}}{(n-j) ! n^{j}} \\
& =-\delta_{k, 1}+\sum_{i=1}^{k-1}(-1)^{k-1-i} C_{k-1, i} n Q_{n}(i)
\end{aligned}
$$

where $\delta_{k, 1}$ is the Kronecker delta. Therefore

$$
Q_{n}(k)=n\left[\sum_{i=0}^{k-2}(-1)^{k-i} C_{k-1, i} Q_{n}(i)\right]+\delta_{k, 1} .
$$

The value of $Q_{m}(0)$ is $\left(n ! e^{n} / n^{n+1}\right)[1-\gamma(n, n) /(n-1) !]$ where $\gamma(n, n)$ is the incomplete gamma function and can be approximated by (Knuth [3])

$$
\frac{1}{n}\left[\left(\frac{\pi n}{2}\right)^{1 / 2}-\frac{1}{3}+\frac{1}{12}\left(\frac{\pi}{2 n}\right)^{1 / 2}-\frac{91}{540 n}+\frac{1}{288}\left(\frac{\pi}{2 n^{3}}\right)^{1 / 2}+O\left(n^{-2}\right)\right]
$$

Further, we have from the recurrence relation that $Q_{n}(1)=\delta_{1,1}=1$ and $Q_{n}(2)$ $=n Q_{n}(0)$.

Now $Q_{n}(k)$ is a polynomial in $n$ plus $Q_{n}(0)$ times a polynomial in $n$. For large $n$, $Q_{n}(k)$ can be approximated by its leading term; i.e. letting

$$
\begin{aligned}
a_{n, k} & =1 \cdot 3 \cdot 5 \cdots(k-1) n^{k / 2} Q_{n}(0) & & \text { if } k \text { is even, } \\
& =2 \cdot 4 \cdot 6 \cdots(k-1) n^{(k-1) / 2} & & \text { if } k \text { is odd, }
\end{aligned}
$$

then

$$
Q_{n}(k)=a_{n, k}+o\left(n^{(k-1) / 2}\right) .
$$

Collecting together the various results for large $n$ we have:

$$
\begin{array}{rlrl}
E_{F_{n}, 1}(l) & =G_{1,1}\left(\frac{\pi n}{2}\right)^{1 / 2}+\frac{1}{6}+o(1) & \\
E_{F_{n}, r}\left(l^{m}\right) & =(1 \cdot 3 \cdot 5 \cdots m)\left(\frac{\pi}{2}\right)^{1 / 2} G_{r, m} n^{m / 2}+o\left(n^{m / 2}\right) & & \text { for } m \text { odd } \\
& =(2 \cdot 4 \cdot 6 \cdots m) G_{r, m} n^{m / 2}+o\left(n^{m / 2}\right) & \text { for } m \text { even. }
\end{array}
$$


Using Shepp and Lloyd's [2] results for moments of shortest cycles one can also show that

$$
E_{F_{n}, r}(s)=S_{r, 1} Q_{n}(1, r)+o\left(Q_{n}(1, r)\right)
$$

and

$$
E_{F_{n}, r}\left(s^{m}\right)=S_{r, m} Q_{n}(m, r-1)+o\left(Q_{n}(m, r-1)\right) \text { for } m>2,
$$

where $E_{F_{n}, r}\left(s^{m}\right)$ is the expected value of the $m$ th moment of the $r$ th shortest cycle, $S_{r, m}$ is a coefficient given in Shepp and Lloyd [2], and

$$
Q_{n}(m, r)=\sum_{j=1}^{n} \frac{(n-1) ! j^{m}(\log j)^{r}}{(n-j) ! n^{j}}
$$

We have not found any asymptotic results for this sum when $r \neq 0$.

For values of $n$ from 1 to 50 we compared the actual average length of the longest cycle to that predicted by the formula

$$
l_{\text {ave }}=.7824816 n^{1 / 2}+.104055+.0652068 n^{-1 / 2}-.1052117 n^{-1}+.0416667 n^{-3 / 2}
$$

obtained by taking the first five terms in the expansion of $G_{1,1} Q_{n}(2)+1 / 6$. For $n \leqq 5$ the formula gave too low an answer. For $6 \leqq n \leqq 50$ the formula gave too high an answer, with the maximum error of .00895 at $n=24$. Above $n=24$ the error slowly decreased to .00808 at $n=50$.

\section{REFERENCES}

1. D. E. Knuth, The art of computer programming, Vol. 2, Addison-Wesley, Reading, Mass. (to appear).

2. L. A. Shepp and S. P. Lloyd, Ordered cycle length in a random permutation, Trans. Amer. Math. Soc. 121 (1966), 340-357.

3. D. E. Knuth, The art of computer programming, Vol. 1, Addison-Wesley, Reading, Mass., 1968, p., 117.

University of Wisconsin, Computer Sciences Department, Madison, Wisconsin 